\title{
Formation Mechanisms of ZnO Spherulites and Derivatives
}

\author{
Bethany M. Connolly, ${ }^{\dagger}$ Heather F. Greer ${ }^{\dagger}$ and Wuzong Zhou* \\ EaStCHEM, School of Chemistry, University of St Andrews, St Andrews, Fife KY16 9ST, United Kingdom
}

\begin{abstract}
ZnO microparticles have been solvothermally synthesized from zinc acetate and formic acid. The resulting microspherulites consist of radially arranged nanorods which undergo a range of re-crystallisation pathways to produce particles with different terminal morphologies. Crystallisation on the particle surface results in hexagonal microdisc decorated hierarchical microspheres. These particles expose only the (ooo1) crystallographic plane of $\mathrm{ZnO}$ and further develop into a sea-urchin like morphology, when the microdiscs grow up along the [ooor] direction into hexagonal microrods. Alternatively, crystallisation of the particle core into a $\mathrm{ZnO}$ plate, facilitates asymmetric assembly of nanocrystallites, forming a hexagonal cone on one side of the plate. The particle has a mushroom-like terminal morphology. When simultaneous surface and core re-crystallisation takes place, wood gyro-shaped particles are observed, in which all the surface discs face a single direction. The microstructures of the particles were investigated using XRD, electron microscopy and surface colouring with adsorbed charged dyes. Structural studies of the early growth stages and subsequent morphology evolution support a dipole field driven formation mechanism. This work offers an improved understanding of the formation of other spherulites, in particular, naturally occurring mineral calcium carbonate spherulites.
\end{abstract}

\section{INTRODUCTION}

Highly ordered nanomaterials, especially those with selectively exposed facets, exhibit unique physical properties not otherwise observed in the bulk phase. A greater understanding of nanomorphology formation mechanisms is needed if finely tuned materials are to be designed for targeted applications.

$\mathrm{ZnO}$ wurtzite is one such promising material, having generated significant research interest due to its desirable material characteristics e.g. low cost, non-toxicity, wide band gap $(3.37 \mathrm{eV})$ and large exciton binding energy (6o $\mathrm{meV}$ at room temperature). ${ }^{1}$ Electrical, catalytic and optoelectronic properties, combined with thermal and chemical stability, make $\mathrm{ZnO}$ an ideal material in many applications including gas sensors, ${ }^{2}$ solar cells, ${ }^{3} \mathrm{UV}$ lasers ${ }^{4}$ and cell labels. ${ }^{5}$ The extensive morphological diversity exhibited by this material allows synthetical control of nanostructures with finely-tuned properties for specific applications.

Preparation of bulk, thin-film and nanoparticle $\mathrm{ZnO}$ structures have been reported through a number of synthetic methods including hydro-/solvo- thermal syntheses, ${ }^{6,7}$ sol-gel synthesis ${ }^{8}$ and metal-organic chemical vapor deposition (MOCVD) ${ }^{9}$ etc. Compared to high temperature $\left(>450{ }^{\circ} \mathrm{C}\right)$ vapor phase methods, solution-based synthetic methods conducted at low temperatures $\left(25^{-200}{ }^{\circ} \mathrm{C}\right)$ have attracted considerable attention due to their simplicity, low cost and ability to generate morphologically homogeneous materials with high product yield. Significantly, many unusual $\mathrm{ZnO}$ morphologies have been fabricated, ranging from simple discs, ${ }^{10}$ rods and tubes ${ }^{11}$ to complex twin-crystals, ${ }^{12,13}$ hierarchical secondary and tertiary branched hexagonal rods,,${ }^{14}$ bowls,${ }^{15}$ hexapods,${ }^{16}$ and hollow microspheres. ${ }^{17}$ If these various crystal morphologies are to be synthesized with high experimental control for target applications, the formation mechanisms must be fully understood. Fundamental knowledge of both classical and non-classical nucleation and crystallisation theories is therefore needed. ${ }^{18}$

Classical crystallisation, defined as nucleation in a supersaturated solution followed by layer-by-layer deposition of the building units, is often insufficient to explain the construction of complex particle morphologies. Non-classical pathways include reversed crystal growth ${ }^{19,20}$ and particle mediated processes (where alignment is controlled by oriented attachment, face selective molecules, physical forces, etc.) have become increasingly common. ${ }^{21}$ The spontaneous assembly of nanoparticles into that with common crystallographic orientations is thought to be thermodynamically driven; surface free energy is reduced by elimination of high energy surfaces through adjacent particle fusing. Electric ${ }^{22}$ and magnetic fields, ${ }^{23,24}$ dipole forces ${ }^{25,26}$ and interactions between surface adsorbents ${ }^{27}$ have also been proposed as the driving force for nanoparticle alignment along specific crystallographic orientations. 
To modulate and therefore control the building and ordering of $\mathrm{ZnO}$ superstructures, considerable synthetic control of both particle size and exposure of desirable facets is needed. This can be achieved through the inclusion of organic additives e.g. capping agents and surfactants in the synthetic method. ${ }^{28,29}$ Wurtzite $\mathrm{ZnO}$ is comprised of alternating planes of tetrahedrally coordinated $\mathrm{Zn}^{2+}$ and $\mathrm{O}^{2-}$ ions, the result of which is an intrinsic dipole moment along the crystallographic c-axis. The presence of $\mathrm{Zn}^{2+}$ and an $\mathrm{O}^{2-}$ terminated faces in $\mathrm{ZnO}$ crystals results in the exposure of both polar $\{0001\}$ and semi-polar $\{10 \overline{1} 1\}$ facets in certain morphologies e.g. hexagonal prisms ${ }^{30}$ and nanopyramids. ${ }^{29}$ The otherwise high surface energy of these planes can be reduced by selective capping using appropriate organic molecules. Theoretical and experimental studies have shown that $\{0001\}$ facets of $\mathrm{ZnO}^{31}$ are extraordinarily reactive compared to other crystal facets. Engineering of the surface structure in the micro- or nano-scale to expose a large percentage of $\{0001\}$ facets has been reported for $\mathrm{ZnO}$. $^{22-35}$

In the present work, the detailed formation processes of both $\mathrm{ZnO}$ spherulites and its derivative morphologies including disc decorated microspheres, sea urchin-like, mushroom-like and wood gyro-like microparticles were investigated. For the construction of spherulites, nucleation and development of $\mathrm{ZnO}$ nanocrystallites must take place inside a gel like organic substrate. The intrinsic dipole field in the crystallites plays an important role in the orientated self-assembly of these nanoparticles. Different locations of re-crystallisation in the spherulites results in different derived terminal morphologies.

\section{EXPERIMENTAL SECTION}

Preparation of $\mathrm{ZnO}$ specimens. The synthetic method for $\mathrm{ZnO}$ was the same as that previously reported by He et al. ${ }^{36}$ The procedure involved dissolving $1.00 \mathrm{~g}$ zinc acetate dihydrate (4.55 mmol, $98 \%$, from Sigma-Aldrich) in $31 \mathrm{~mL}$ ethanol followed by addition of $0.598 \mathrm{~g}$ formic acid (13 $\mathrm{mmol}, \geqslant 95 \%$, from Sigma-Aldrich). A white precipitate appeared immediately. The suspension was sealed in a 50 $\mathrm{mL}$ Teflon lined stainless steel autoclave and heated at 150 ${ }^{\circ} \mathrm{C}$ for a selected time. The resulting powder product was recovered by centrifugation (300o r.p.m. for $3 \mathrm{~min}$ ) and washed three times with ethanol. The sample was then dried at $60{ }^{\circ} \mathrm{C}$ overnight. Specimens at different growth stages were collected under identical conditions except different thermal reaction times of o h, $1 \mathrm{~h}, 2 \mathrm{~h}, 3 \mathrm{~h}, 24 \mathrm{~h}, 45 \mathrm{~h}$ and $168 \mathrm{~h}$, were applied.

Surface colouring using charged dyes. Safranin $\mathrm{T}$ and congo red dyes were obtained from Sigma-Aldrich and used without further purification. $\mathrm{ZnO}$ precipitate were immersed in a standard dye solution ( $0.5 \mathrm{~g} / \mathrm{L})$ prepared with deionized water, vigorously shaken and left to stand overnight. The precipitate was washed three times with distilled water before placing on a glass microscope slide.
After drying at $60^{\circ} \mathrm{C}$, the $\mathrm{ZnO}$ particles were observed by optical microscopy.

Specimen characterization. Powder X-ray diffraction (PXRD) was performed on a PANalytical Empyrean diffractometer, using $\mathrm{Cu} \mathrm{K}_{\alpha}$ radiation and operated in reflectance mode. Analysis of the PXRD patterns was carried out using Highscore plus software. Scanning electron microscopic (SEM) images of the specimens were obtained using a JEOL JSM-67ooF microscope or a TESCAN MIRA3, operating at $5 \mathrm{kV}$. Specimens were sputter coated with a thin $\mathrm{Au}$ film using a Quorum Technologies Q150R ES sputter coater/carbon coater, in order to prevent the sample from charging. Transmission electron microscopic (TEM) images and selected area electron diffraction (SAED) patterns were attained using either a JEOL JEM-2o11 or a FEI Titan Themis 200 microscope operating at an accelerating voltage of $200 \mathrm{kV}$. The JEOL JEM-2011, fitted with a $\mathrm{LaB}_{6}$ filament is equipped with an Oxford Instruments Link ISIS SemiSTEM EDX system and a Gatan 794 CCD camera. The FEI Titan Themis $200 \mathrm{~S} / \mathrm{TEM}$ is fitted with an X-FEG gun and equipped with FEI's Super-X windowless EDX detector comprising of quad silicon drift detectors (SDD). TEM and high resolution TEM (HRTEM) images were recorded using a FEI Ceta $4 \mathrm{k} \times 4 \mathrm{k}$ CMOS camera. The cross section SEM images of particles were recorded on FEI Scios Dualbeam microscope operated at $5 \mathrm{kV}$ and using a gallium liquid-metal ion source in focused ion beam (FIB). Optical microscopic images were recorded using an Olympus BHM series metallurgical microscope system and a Canon Power Shot G6 digital camera.

\section{RESULTS AND DISCUSSION}

In the present synthesis, four typical morphologies of $\mathrm{ZnO}$ particles were observed by SEM (Figure 1), i.e. (a) mushroom shape, (b) disc decorated sphere, (c) sea urchin-like shape and (d) wood gyro-like shape. Despite extensive differences in morphology, these particles were observed to co-exist within samples. Although the synthetic method previously reported by He et al. ${ }^{36}$ was utilised in the present work, only the disc decorated spheres have been previously reported. The remaining three types of particles were newly observed in terms of $\mathrm{ZnO}$ morphology.

To understand the formation mechanisms of these particles, it is necessary to examine the microstructures of early stage specimens. Primary $\mathrm{ZnO}$ nanocrystals were observed before the formation of the above-mentioned morphologies. XRD patterns (Figure 2) of three early stage samples prepared from $0-1.5 \mathrm{~h}$ confirmed the time scale of the phase change in the reaction mixture. The XRD pattern of the white precipitate collected prior to thermal treatment (Figure 2a) was indexed to a pure phase of monoclinic zinc formate (JCPDS card no. o1-076-8283) with the unit cell parameters of $a=11.33, b=9.84, c=18.17 \AA, \beta=127.27^{\circ}$, space group $\mathrm{P}_{2} / \mathrm{c}$. However, after $1 \mathrm{~h}$ of thermal treatment, hexagonal wurtzite $\mathrm{ZnO}$ (JCPDS card no. 70-2551) with the unit cell parameters of $a=3.249, c=5.207 \AA$, space group 
$\mathrm{P}_{3} \mathrm{mc}$ was the dominant crystalline phase. At this time, low intensity reflections corresponding to zinc formate remained (indicated by an arrow in Figure $2 \mathrm{~b}$ ) demonstrating that the reaction had not yet reached completion. A pure $\mathrm{ZnO}$ phase was obtained after reaction times of $1.5 \mathrm{~h}$ (Figure 2c) or longer.
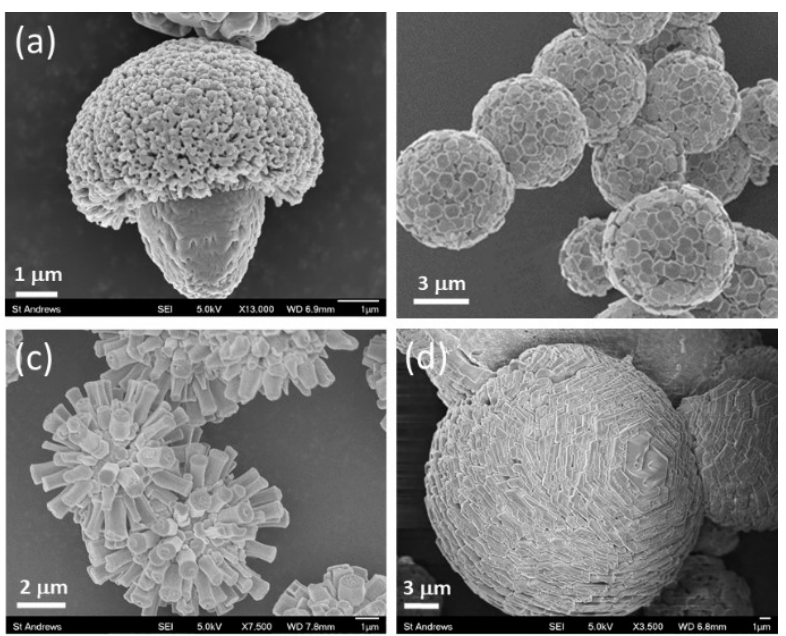

Figure 1. SEM images of different morphologies of $\mathrm{ZnO}$, (a) mushroom-shape from $45 \mathrm{~h}$ sample, (b) disc decorated spheres from $2 \mathrm{~h}$ sample. (c) Sea urchin shaped and (d) wood gyro shaped spheres from $24 \mathrm{~h}$ sample.

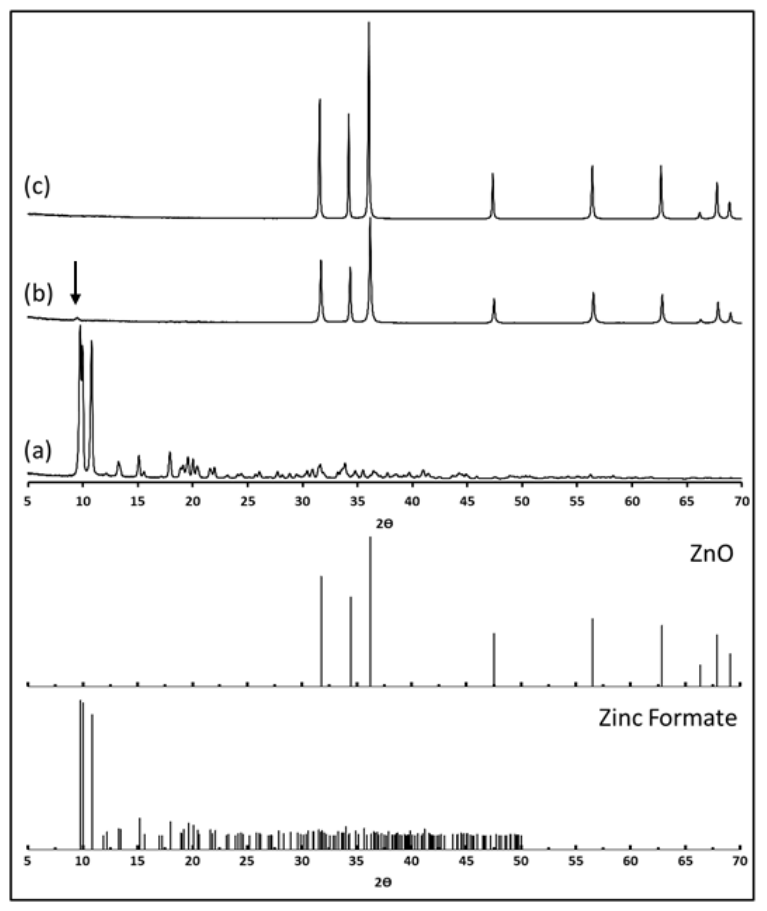

Figure 2. XRD patterns of specimens prepared with reaction times of (a) o h, (b) $1 \mathrm{~h}$ and (c) $1.5 \mathrm{~h}$. Reference patterns for zinc formate and $\mathrm{ZnO}$ are also shown.
The XRD data supports the chemical reactions reported by He et al. ${ }^{36}$ which are as follows:

$$
\begin{aligned}
& \mathrm{Zn}\left(\mathrm{CH}_{3} \mathrm{COO}\right)_{2} \cdot 2 \mathrm{H}_{2} \mathrm{O}+2 \mathrm{HCOOH} \rightarrow \\
& \mathrm{Zn}(\mathrm{COOH})_{2} \cdot 2 \mathrm{H}_{2} \mathrm{O}+{ }_{2} \mathrm{CH}_{3} \mathrm{COOH}
\end{aligned}
$$

$$
\begin{aligned}
& \mathrm{Zn}(\mathrm{COOH})_{2} \cdot 2 \mathrm{H}_{2} \mathrm{O}+2 \mathrm{CH}_{3} \mathrm{CH}_{2} \mathrm{OH} \rightarrow \\
& \mathrm{ZnO}+2 \mathrm{HCOOCH}_{2} \mathrm{CH}_{3}+3 \mathrm{H}_{2} \mathrm{O}
\end{aligned}
$$

The reaction between zinc acetate dihydrate and formic acid spontaneously yields zinc formate dihydrate crystals prior to solvothermal treatment (eq. 1). Zinc formate has a low solubility in ethanol but undergoes complete conversion to $\mathrm{ZnO}$ within $1.5 \mathrm{~h}$ of heating at $150^{\circ} \mathrm{C}$ via Fischer esterification (eq. 2).

To further elucidate the crystal growth mechanism, we examined solid samples produced over a range of reaction times. As previously discussed, mixing of the chemical precursors resulted in immediate precipitation of pure phase of zinc formate dihydrate, as detected by XRD (Figure 2a). SEM and TEM images revealed aggregates of $50 \mathrm{~nm}$ zinc formate dihydrate nanocrystallites (Figure $\mathrm{S} 1$, Supporting Information, SI).
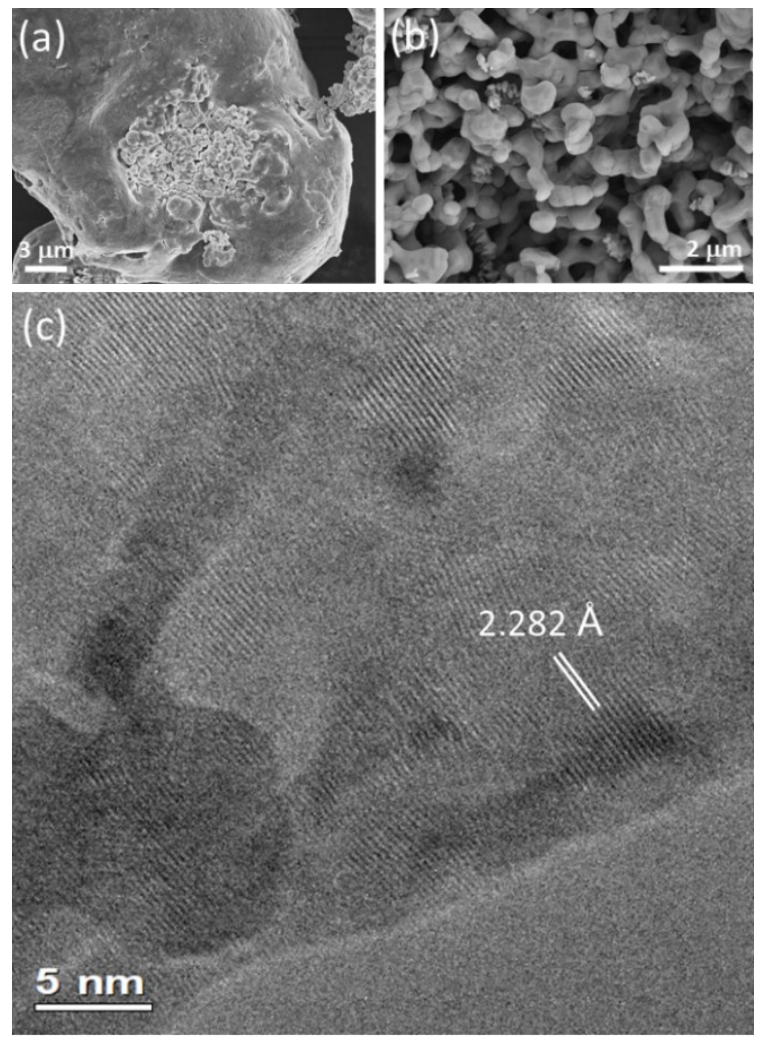

Figure 3. SEM images of $1 \mathrm{~h}$ sample, (a) before and (b) after washing with ethanol. (c) HRTEM image of part of soft matter substrate showing $\mathrm{ZnO}$ nanocrystallites. The corresponding low magnification TEM image is shown in Figure S2 in SI. 
The zinc formate dihydrate nanocrystals were solvothermally converted into $\mathrm{ZnO}$ within $1.5 \mathrm{~h}$. SEM images of a sample obtained after a $1 \mathrm{~h}$ reaction time show that the assynthesized specimen is comprised of small particles embedded in a gel-like soft substrate (Figure 3a). After washing with ethanol, the loose soft substrate can be completely removed, allowing previously enclosed small particles to be observed. However, most particles do not look like pure $\mathrm{ZnO}$, but are covered by the soft substance or form an inorganic and organic composite (Figure $3 \mathrm{~b}$ ). The particles are much larger than the nanocrystallites as revealed by HRTEM and connected each other to form a network.

TEM analysis of the soft matter substrate confirms polymer like features, as shown by Figure S2 (SI). Further HRTEM imaging of this soft matter (Figure $3 \mathrm{c}$ ) reveals the presence of additional smaller nanocrystallites (ca. $5 \mathrm{~nm}$ in diameter) separately embedded within. The measured $d-$ spacings from these nanocrystallites $(2.282 \AA)$ correspond to the $\{10 \overline{1} \mathrm{o}\}$ planes of $\mathrm{ZnO}$.

The above observation suggests that both nucleation and subsequent crystal growth of $\mathrm{ZnO}$ nanocrystallites takes place within the soft substrate. The soft substrate may act as a suppressor of growth of individual crystals by limiting diffusion of reactants dissolved in the surrounding solution.

Since all the organic molecules used in the system are soluble in ethanol, the formation of the gel like soft substance must be due to formation of larger molecules or polymerization of the organic molecules, probably enhanced by embedded inorganic ions. Water released during the reaction (see eq. 2) may also reduce the solubility of ethyl formate.

Spherulites. The nanocrystallites inside the soft substrate underwent subsequent self-assembly into spherical particles. Figure 4a shows a representative cluster of assembled nanocrystallites in the $\mathrm{i} \mathrm{h}$ sample after washing. Inside the network of inorganic/organic composite, there are some spherical particles. It is interesting to see cross sections of three spherical particles with their $1-3 \mu \mathrm{m}$ sized core removed during washing, and very short nanorods gathering on the surface of the cores in a radial arrangement (inset of Figure 4a).

The resulting nanorods were observed to grow to ca. several microns in length, leading to the formation of highly symmetric spherulites as shown in Figure $4 \mathrm{~b}$. Observation of a broken spherulite (Figure 4c) enables us to see that the particle consists of long nanorods ( $5 \mu \mathrm{m}$ in length) in a radial arrangement on the surface of a spherical core $(3 \mu \mathrm{m}$ in diameter), which has been washed away, leaving a spherical cage. The original core is expected to mainly contain organic substance.

The whole growth process of such spherulites appears to take place inside the soft substrate with the inter-nanorod space also filled by this organic material. Even samples extensively washed prior to SEM and TEM imaging exhibited residual soft substrate in the inter-nanorod spaces. Figure
S3a in SI shows two particles, a spherulite and a disc decorated sphere, linked by a bridge of residual soft substance, while a broken spherulite in Figure S3b in SI shows internalised soft substance.
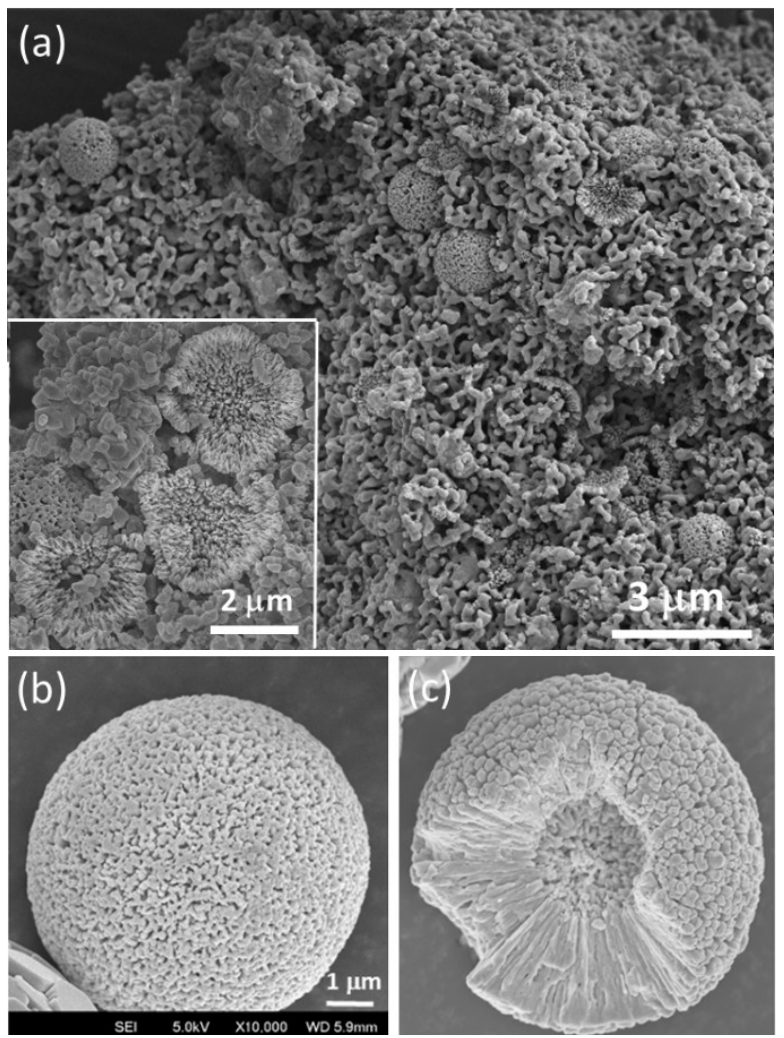

Figure 4. (a) SEM image of $\mathrm{l}$ h sample after washing with ethanol, showing a network of inorganic/organic composite. The inset is an enlarged image showing that very short nanorods start to gather on the surface of spherical cores with a diameter of about $3 \mu \mathrm{m}$. (b) and (c) SEM images of a whole spherulite and a broken spherulite from a $45 \mathrm{~h}$ sample after washing.

The crystal structure and orientation of the microrods as the principal building units of the spherulites were investigated using TEM. Figure 5a shows two microrods. HRTEM analysis (Figure $5 \mathrm{c}$ ) from the large end of the rod (marked in Figure 5a) and the corresponding SAED pattern (Figure $5 \mathrm{~b}$ ) demonstrate a single crystal structure with the [ooor] zone axis parallel to the long axis of the microrod. The high crystallinity of this end resulted from surface recrystallisation.

On the other hand, the thin end is polycrystalline consisting of oval shaped $\mathrm{ZnO}$ nanocrystallites $(123 \pm 21 \mathrm{~nm}$ by $64 \pm 12 \mathrm{~nm}$ in dimension), which are arranged 'end-to-end' lengthwise in a semi-ordered manner (Figure $\mathrm{S}_{4} \mathrm{a}$ in $\mathrm{SI}$ ). HRTEM confirmed the long axis of the elongated nanoparticles is along the [oool] direction. This further supports that the growth along the c-axis is fast with oriented aggre- 
gation on this direction explained by considering the intrinsic dipole moment associated with $\mathrm{ZnO} .{ }^{37}$ Some oval particles have a domain structure, implying that they formed with assembly of $5 \mathrm{~nm}$ sized nanocrystallites (Figure $\mathrm{S}_{4} \mathrm{~b}$, in SI). The nanocrystallites which comprise the microrods are therefore self-orientated with their c-axis parallel to the radial direction in the spherulites. TEM imaging of a whole spherulite and SAED patterns from different areas of the same particle confirmed such a construction as seen in Figure $\mathrm{S}_{5}$ in SI. These polycrystalline spherical particles expose only one specific crystal plane, the (ooo1) plane.
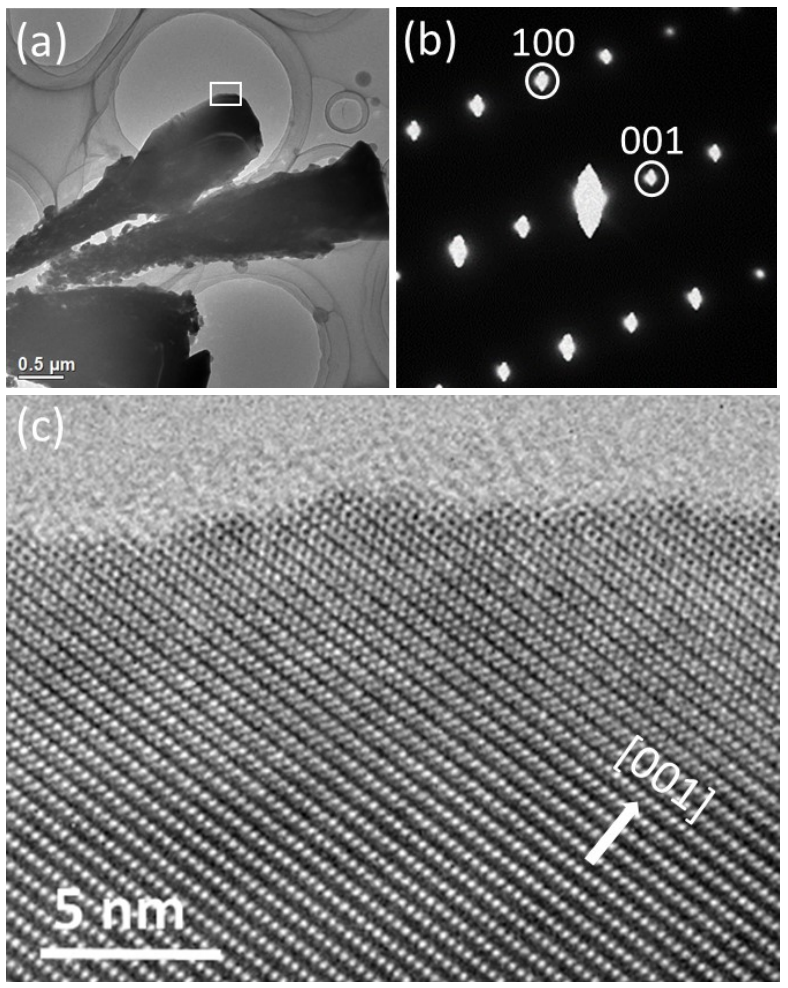

Figure 5. (a) TEM image of individual microrods from a spherulite in $45 \mathrm{~h}$ sample. (b) The corresponding SAED pattern from the top microrod, indexed to the $\mathrm{ZnO}$ structure. (c) HRTEM image recorded from the marked area in (a).

Mushroom-shaped particles. Further crystallisation of the embedded spherulites facilitates core development into a doughnut-shaped plate (Figure 6a). This plate appears to be the origin of the 'stem' in mushroom-like particles. To verify this, FIB-SEM was implemented with the ion beam used to selectively mill away half an asymmetric particle. This allowed visualisation of an early-stage core where a stem was about to extend from (Figure 6c). Interestingly, such a 'stem' only developed on one side of the central plate with a large visible crack around it (Figure 6b). Also apparent is the highly porous nature of the surrounding particle in comparison to the dense nature of the material which occupies the core plate.
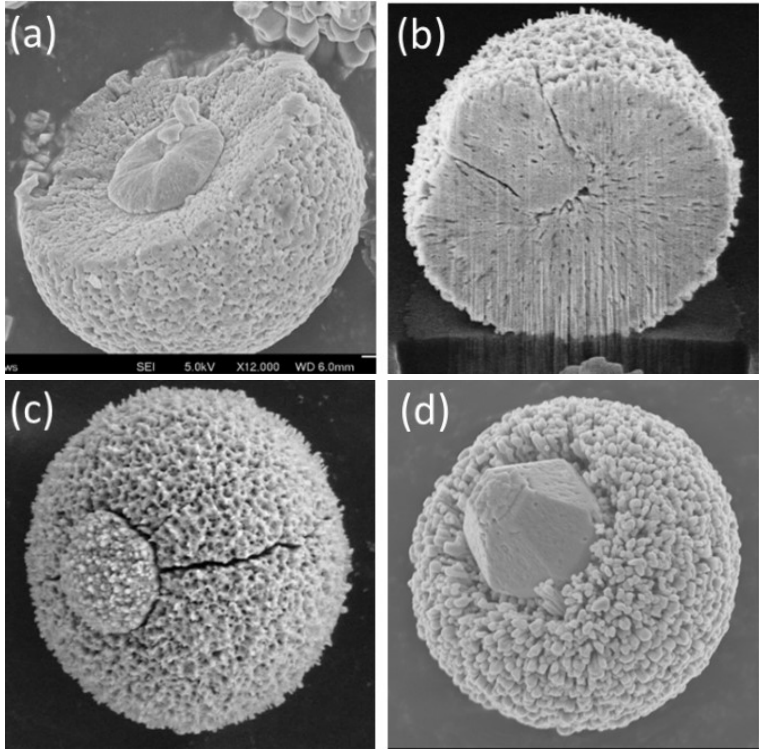

Figure 6. SEM images of $\mathrm{ZnO}$ particles showing morphology evolution from spherulite to a mushroom shape.

This cylindrical component re-crystallises further until it breaks from the surface to form a hexagonal pyramid 'stem'. During the growth of these 'stems', the opposing half of the spherulite remains unchanged. The result of this asymmetric re-crystallisation pathway was a terminal particle morphology reminiscent of a mushroom (Figure $6 \mathrm{~d}$ ). The regular, hexagonal shape of the 'stem' implies formation of a large $\mathrm{ZnO}$ single crystal or a high density self-orientated polycrystalline aggregate. SAED patterns and HRTEM images obtained from a 'stem' reveal a single crystal property, with the c-axis perpendicular to the core plate (Figure S6, in SI).

The mushroom shape of the particles is not an intermediate but a terminal morphology. In samples obtained after $168 \mathrm{~h}$ of solvothermal treatment, numerous mushroomshaped particles were observed (Figure $\mathrm{S}_{7}$, in SI).

Disc decorated spheres. An alternative crystallisation pathway was also observed whereby surface re-crystallisation took place preferentially over core re-crystallisation. This resulted in the formation of hexagonal tiles on the surface of spherulites (Figure $1 b$ ). The occurrence of initial crystallisation at the surface suggests that this was the most reactive/unstable region of the particles. Absence of protective soft substance on such particles may result in surface exposure to the reactants in the solution, increasing the rate of surface re-crystallization. The heterogenous morphologies of particles observed within the same sample may thus stem from the non-homogenous distribution of soft-matter substance within the reaction mixture. As seen in Figure $1 b$, the whole surface of these decorated spheres is covered by hexagonal discs with an average diameter of about $1 \mu \mathrm{m}$. Underneath the surface disc layer, the internal structure remains identical to the primary 
spherulite from which it originated as the core has not yet undergone a re-crystallisation process (Figure S8).

Inward extension of the re-crystallization process results in an increase of disc thickness and subsequent formation of microcones. Growth from polycrystalline to single crystal state can take place in two different ways. Firstly, Ostwald ripening in which smaller particles dissolve and their constituent atoms, molecules or ions deposit on larger particles to reach a more thermodynamically stable state with a minimised surface area to volume ratio. ${ }^{38}$ Alternatively, oriented aggregation can occur in which separate nuclei orient into the same direction before a single crystal forms by fusing at the interfaces. 39 During the surface re-crystallisation of $\mathrm{ZnO}$ particles in the present work, these processes were indistinguishable so more likely took place simultaneously.

Sea urchin shaped particles. Crystal growth of the discs may also occur outwards if the concentration of the precursor molecules in the solution is high enough. Consequently, the hexagonal discs would grow longer into hexagonal microrods as shown in Figure 1c, forming a sea urchin like morphology. On the surface of some other particles, bottle-shaped microrods developed rather than a hexagonal feature (Figure $7 \mathrm{a}$ ). The reason for forming such a novel shape is not understood. It is probably the result of competition between forming hexagonal microrods (Figure 1c) and forming cones (Figure $6 \mathrm{~d}$ ). Figure $7 \mathrm{~b}$ is a naked core of a $\mathrm{ZnO}$ spherical particle when all the microrods have been removed. The core, $\sim 4 \mu \mathrm{m}$ in diameter, seems to be disordered and has no large crystals developed in it. The main component must be organic soft substance.
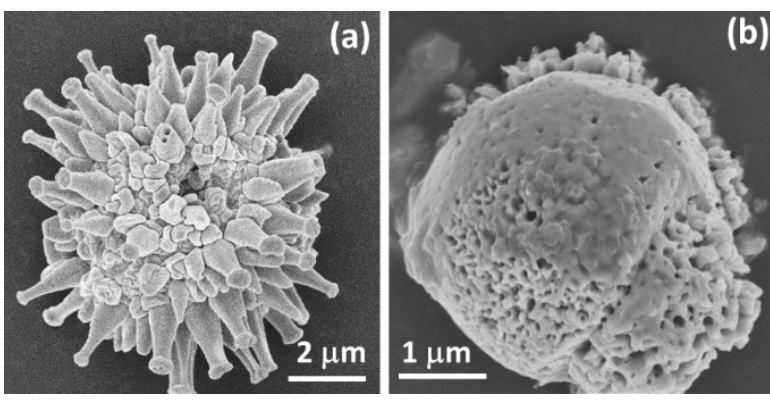

Figure 7. (a) SEM image of a sea urchin like $\mathrm{ZnO}$ particle with bottle-shaped microrods on the surface. (b) SEM image of a core of a $\mathrm{ZnO}$ spherical particle.

Wood gyro like particles. If re-crystallisation of a plate in the spherulite core takes place prior to the particle breaking free of its soft matter surroundings, a wood gyro like particle morphology results (Figure 1d). When the surface of such a particle undergoes re-crystallisation, the discs do not lie parallel to the particle surface but show orientation with all crystallographic c-axis facing almost the same direction. The particles are oriented along a central axis, as indicated by arrows in Figure 8a. The SEM image of a cross section of a spherical particle with the same surface feature shows development of the core crystal (Figure 8b). This crystal grows up only on one side to form a hexagonal rod (Figure 8c). We propose that this orientated arrangement of surface discs is driven by a localised dipole field, originating from the central crystalline plate (Figure 8d).
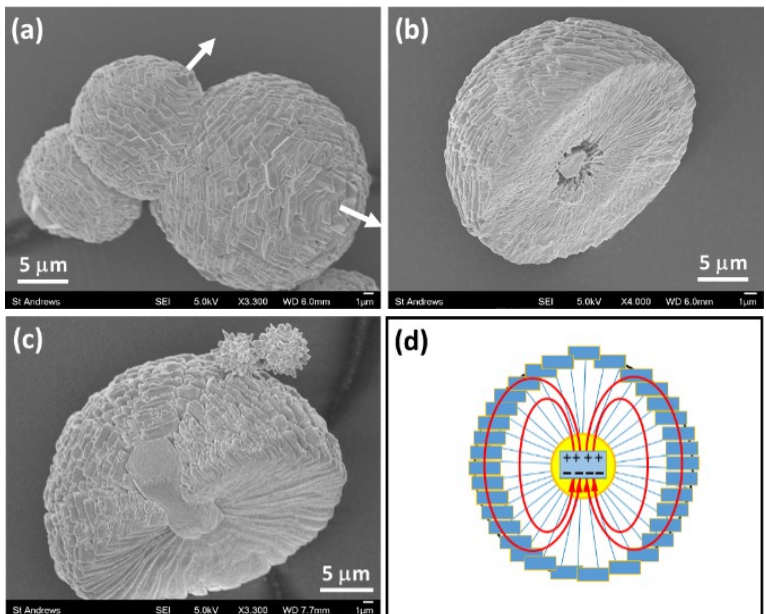

Figure 8. SEM images of some wood gyro shaped particles, (a) particles with central axis indicated by the arrows, (b) a cross section showing a dense core, and (c) half particle showing a hexagonal rod along the central axis. (d) Schematic drawing to elucidate ordering of the surface discs driven by a dipole field from the plate in the core.

The microstructural investigation indicates that all the morphologies of $\mathrm{ZnO}$ produced in the present work are derived from spherulite and governed by the core and surface crystallisation. A consequence of this process is that the surface will only expose either the $\{0001\}$ or $\{000 \overline{1}\}$ planes with the former being positively charged and the latter negative. Charged dyes were utilised to identify the charge of the surface exposed planes on the $\mathrm{ZnO}$ spherulites. Optical microscope images of $\mathrm{CaCO}_{3}{ }^{40}$ and $\mathrm{ZnO}^{25}$ stained with negatively charged dye (Congo Red) and positively charged dye (Safranin T) have previously been reported in support of dipole field directed mechanisms. In the present work, a sample of as produced $\mathrm{ZnO}$ microspheres (after $45 \mathrm{~h}$ growth, containing mainly mushroom-shaped and disc decorated spherical particles) was immersed in standard solutions of 0.5 g/L Congo Red or Safranin T under the assumption that dye with the opposite charge to the particle surface would exhibit better adsorption. The optical microscopic images of unstained and Congo red dye are identical in colour while $\mathrm{ZnO}$ stained with safranin $\mathrm{T}$ shows a much darker contrast (Figure 9). The selective adsorption of Safranin $\mathrm{T}$ and not Congo Red to the particles surface provides strong evidence for a negative surface charge terminated along the $\mathrm{c}$-axis by the $\mathrm{O}^{2-}$ anions.

Mechanisms of morphology evolution. From the results presented above, we are able to propose a mechanistic pathway to rationalize the nucleation, crystal growth and 
morphology evolution of $\mathrm{ZnO}$ spherulites under the conditions in this work. Figure 10 shows the changes step-bystep.

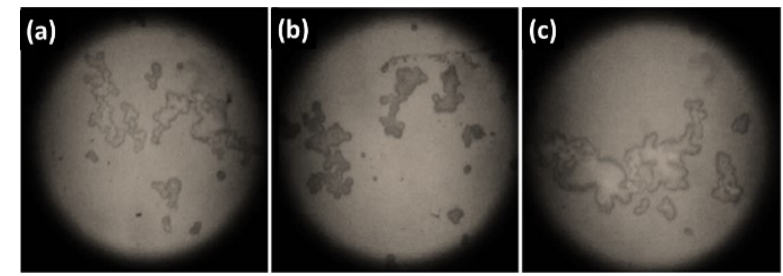

Figure 9. Optical microscope images of $45 \mathrm{~h}$ sample of $\mathrm{ZnO}$ spherical particles stained with (a) anionic congo red, (b) cationic safranin $\mathrm{T}$ dye and (c) no dye. The dark coloured ring encircling each aggregate corresponds to diffraction fringes from the crystalline material.
Step 1. When zinc acetate dihydrate is mixed with formic acid in ethanol, zinc formate dihydrate nanocrystallites $(\sim 50 \mathrm{~nm}$ in diameter) are produced (Figure 10a).

Step 2. Under solvothermal conditions, zinc formate undergoes a phase transformation to zinc oxide nanocrystallites $(5 \mathrm{~nm}$ or larger), releasing ethyl formate and water. The nanocrystallites are embedded in an organic soft substrate (Figure 1ob).

Step 3. In these $\mathrm{ZnO}$ nanocrystallites, nanoscale charge separation between the $\mathrm{Zn}^{2+}$ terminated (oool) surface and $\mathrm{O}^{2-}$ terminated (ooo $\left.\overline{1}\right)$ surface results in a dipole moment. The dipole field interaction which exists between the small nanocrystals is significant and enhances self-orientation and self-assembly of the separated nanocrystallites. Obviously, the property of the organic soft substance is crucial. It should be dense enough to prevent the nanocrystallites from fast growth into large crystals. It should also soft enough, allowing the nanocrystallites to rotate and shift locally.
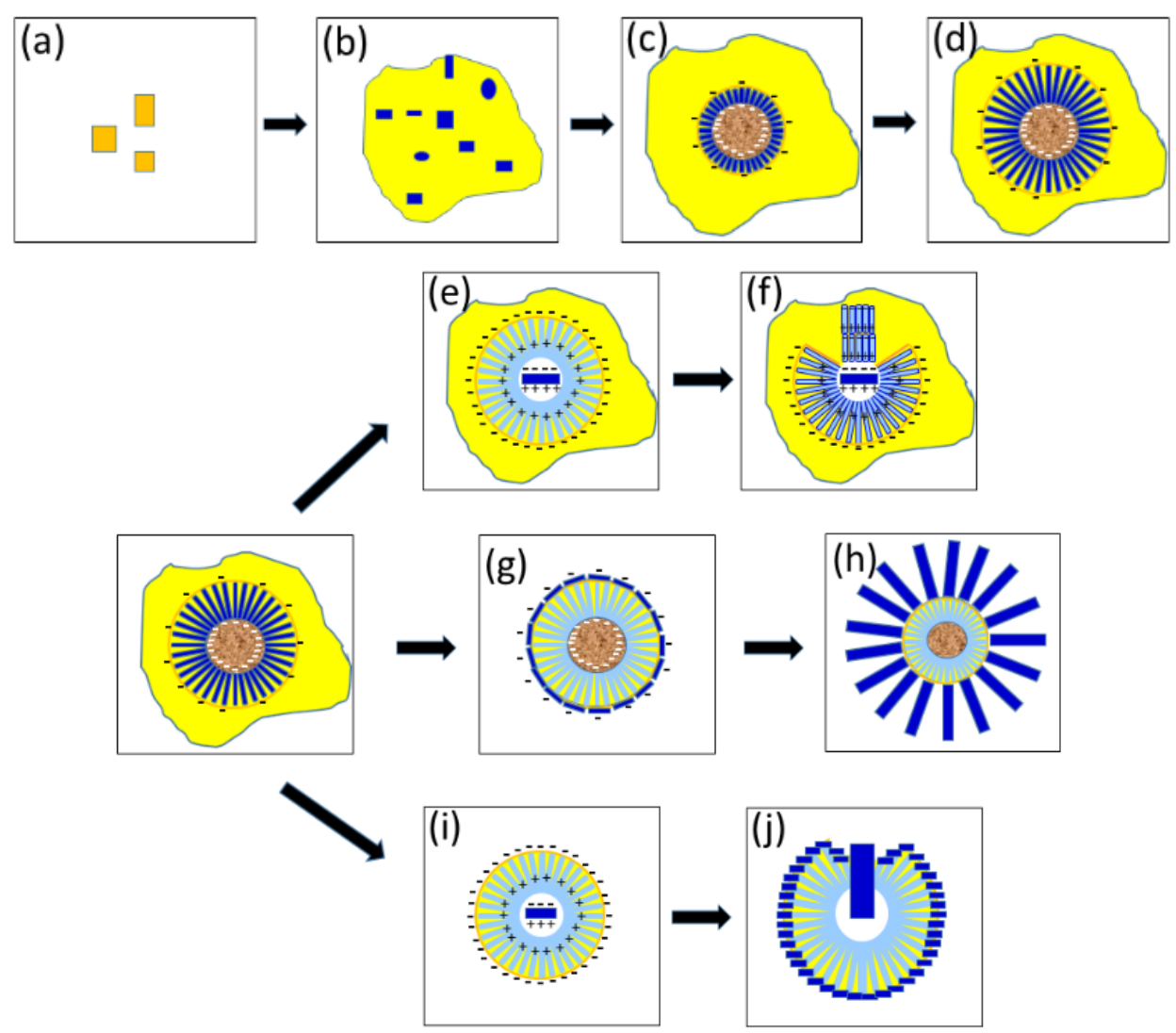

Figure 10. Schematic diagram representing the formation mechanisms of different morphologies of $\mathrm{ZnO}$ particles. Formation of (a) zinc formate nanocrystallites, which undergo phase transformation to form (b) $\mathrm{ZnO}$ nanocrystallites in a soft substrate. (c) Aggregation of $\mathrm{ZnO}$ nanorods on a negatively charged core, forming (d) a spherulite. Route 1 : When the surface of a spherulite is well protected by the soft substrate, (e) a ZnO plate develops in a core, leading to a re-arrangement of nanorods on the negatively charged surface of the central plate to form (f) a mushroom shaped particle. Route 2: When a spherulite exposes to the solution before the formation of the central plate, discs developed due to surface re-crystallization, forming (g) a disc decorated sphere. Further crystal growth of the discs leads to the formation of (h) a sea urchin shaped particle. Route 3: When a spherulite exposes to the solution after the formation of (i) a $\mathrm{ZnO}$ plate in the core, surface discs develop with their orientations following the dipole field force lines generated by the central plate, resulting in (j) a wood gyro shaped particle. 
In the present work, the soft substance consists of $\mathrm{Zn}$ containing precursor and polymerized organic molecules, including acetate, formic acid and ethanol. The nanocrystallites can even migrate through the soft substance to aggregate, forming some $\mathrm{Zn}$ rich spherical particles. These particles have an inorganic/organic composite core, 2 to 3 $\mu \mathrm{m}$ in diameter, which are negatively charged mainly due to a large amount of organic anions. $\mathrm{ZnO}$ nanocrystallites then deposit on the core, aligning radially with their positive sides touching the surface of the core. Therefore, the outer surface of the spherical particles should be negatively charged (Figure 10c).

Step 4. The subsequent attractive and repulsive Coulombic forces between oppositely charged faces can generate an oriented alignment of nanocrystallites along the caxis, forming long nanorods in a radial arrangement. This leads to the formation of 7 - $10 \mu \mathrm{m}$ diameter spherulites (Figure 1od). The particle surface is negatively charged, exposing the $\{000 \overline{1}\}$ plane. More complex morphologies are then derived from these primary spherulites via three recrystallisation pathways, depending on different locations of the particles.

When the surface of a spherulite is well protected all the time by the soft substrate, the morphology evolution follows route 1 . Surface re-crystallisation of the spherulite is prevented by the surrounding organic substrate. Instead, the core may preferentially undergo re-crystallisation to form a $\mathrm{ZnO}$ plate. This large plate exerts a strong dipole field (Figure 10e) over the nearby nanocrystallites.

A similar vaterite plate was found in the center of polycrystalline microspherulites. In that case a gelatin layer sandwiched by two vaterite layers together with the resulting mirror symmetric electric field (both outer surfaces are negatively charged) yielding a twin cauliflower like morphology..$^{\circ}$ In the present work, the central plate is a single layer of $\mathrm{ZnO}$, with charge separation occurring at the two outer surfaces of the crystal. Therefore, the dipole dependent morphology evolution is asymmetric.

In the next step, the constituent nanocrystallites which comprise the spherulite have their inner surface positively charged and their outer surface negatively charged. The charge separation enforced by the presence of the negatively charged core now leads to a repulsive force between the positively charged side of the plate and the surrounding nanocrystallites. Conversely, the negative side of the plate attracts the nanoparticles, which align in a parallel manner to form a dense stem. The result of this asymmetric interaction is the formation of particles resembling a mushroom shape (Figure 1of). These particles also need protection by the soft substance, so that no larger crystals would develop on the surface.

If a spherulite migrates out of the soft substrate exposing to the solution, the surface now becomes available for recrystallisation. The morphology evolution occurs via rout 2. As a consequence, the particle surface is completely covered by one or two layers of hexagonal discs, the so-called disc decorated spheres (Figure $10 \mathrm{~g}$ ). The crystallisation can extend inwards to the particle centre via an Ostwald ripening process, changing discs to cones.

When the growth time increases, the discs on the particle surface can further grow outwards, forming a sea urchin like particles (Figure 10h). This can only happen when the particles are in the solution as free particles and the growth time is long.

In route 3 , a single crystal plate develops in the particle core prior liberation from the soft substrate to the solution (Figure 10i), the formation of surface discs takes place in a new arrangement in which their c-axis is aligned along the dipole field force lines generated by the core (as shown in Figure $8 \mathrm{~d}$ ). The central plate can continue to recrystallise outwards into a hexagonal rod, the result of which is a particle resembling a wood gyro (Figure 10j). The reason for asymmetric growth of the hexagonal rod is the same as that of hexagonal cone in mushroom-shaped particles. Since the particles are now in the solution, a high crystallinity of the hexagonal rods is achieved much quicker.

\section{CONCLUSIONS}

$\mathrm{ZnO}$ nanocrystallites can form inside a soft substrate and aggregate into spherulites with several micrometers in diameter. The dipole field of $\mathrm{ZnO}$ crystals plays an important role in the aggregation and the ordering in the spherulites. On the other hand, the property of the soft substrate is also important, preventing further nanocrystal growth while permitting nanocrystallites rotation and orientation. This is comparable to the formation of biomineral spherulites of $\mathrm{CaCO}_{3}$, where the soft substrate is a biological substance. ${ }^{41}$

All other morphologies observed in the present work can be regarded as derivatives of the primary spherulites. The growth of mushroom-shaped particles without surface recrystallisation is possible when the particles are embedded in a soft substrate. The asymmetric growth of the stem originates from the asymmetric charge separation of a plate in the core. Growth of both disc decorated spheres and sea urchin like particles occurs when spherulites move from the soft substrate to the solution and therefore lack surface protection by the soft substrate. If a $\mathrm{ZnO}$ plate forms first and the discs form later via surface re-crystallization, the orientation of the discs will be affected by the dipole field generated by the central plate, leading to a wood gyro-like shape. The majority of the exposed surface of $\mathrm{ZnO}$ particles discussed herein, except sea urchin like ones, is the (ooo $\overline{1})$ plane. We expect that this research sheds light on the formation mechanism of other morphologies of many metal oxides.

\section{ASSOCIATED CONTENT}

Supporting Information

The Supporting Information is available free of charge on the ACS Publications website at DOI: 
Figure S1, SEM and TEM images of zinc formate nanocrystallites. Figure $\mathrm{S}_{2}$, TEM image of soft substrate from $1 \mathrm{~h}$ sample. Figure $\mathrm{S}_{3}$, SEM images of spherulites containing soft substrate. Figure S4, HRTEM of oval particles in the microrods. Figure $\mathrm{S}_{5}$, TEM image of a whole spherulite with SAED patterns from different parts. Figure S6, TEM and HRTEM images of a stem in a mushroom shaped particle. Figure $\mathrm{S}_{7}$, SEM image of mushroom-shaped particles from $168 \mathrm{~h}$ sample. Figure S8, SEM images of the inner structure of a disc decorated sphere and spherical particles linked by soft substrate.

\section{AUTHOR INFORMATION}

\section{Corresponding Author}

*E-mail: wzhou@st-andrews.ac.uk

ORCID

Wuzong Zhou: 0000-0001-9752-7076

\section{Current Address}

$\dagger$ Department of Chemistry, University of Cambridge, Lensfield Road, Cambridge CB2 1EW, UK.

\section{Notes}

The authors declare no competing financial interest.

\section{ACKNOWLEDGMENT}

B.M.C. would like to thank David Miller and Ross Blackley for their help in operation of electron microscopes. We also thank EPSRC for a platform grant (EP/Ko15540/1) and a Capital Equipment Grant EP/Lo170o8/1.

\section{REFERENCES}

(1) Janotti, A.; Van de Walle, C. G. Fundamentals of zinc oxide as a semiconductor. Rep. Prog. Phys. 2009, 72, No. 126501.

(2) Zhu, L.; Zeng, W. Room-temperature gas sensing of $\mathrm{ZnO}$ based gas sensor: A review. Sens. Actuators A 2017, 267, 242-261.

(3) Saito, M.; Fujihara, S. Large photocurrent generation in dyesensitized ZnO solar cells. Energy Environ. Sci. 20o8, 1, 280-283.

(4) Dong, H.; Liu, Y.; Lu, J.; Chen, Z.; Wang, J.; Zhang, L. Singlecrystalline tower-like $\mathrm{ZnO}$ microrod UV lasers. J. Mater. Chem. C 2013, 1, 202-206.

(5) Gupta, J.; Bhargava, P.; Bahadur, D. Fluorescent ZnO for imaging and induction of DNA fragmentation and ROS-mediated apoptosis in cancer cells. J. Mater. Chem. B 2015, 3, 1968-1978.

(6) Kuo, C.-L.; Kuo, T.-J.; Huang, M. H. Hydrothermal synthesis of $\mathrm{ZnO}$ microspheres and hexagonal microrods with sheetlike and platelike nanostructures. J. Phys. Chem. B, 2005, 109, 20115-20121.

(7) Šarič, A.; Štefanić, G.; Dražić, G.; Gotić, M. Solvothermal synthesis of zinc oxide microspheres. J. Alloys Compd. 2015, 652, 91-99.

(8) Khan, M. F.; Ansari, A. H.; Hameedullah, M.; Ahmad, E.; Husain, F. M.; Zia, Q.; Baig, U.; Zaheer, M. R.; Alam, M. M.; Khan, A. M.; AlOthman, Z. A.; Ahmad, I.; Ashraf, G. Md; Aliev, G. Solgel synthesis of thorn-like $\mathrm{ZnO}$ nanoparticles endorsing mechanical stirring effect and their antimicrobial activities: Potential role as nano-antibiotics. Sci. Rep. 2016, 6, No. 27689.

(9) Park, J. Y.; Lee, D. J.; Kim, S. S. Size control of ZnO nanorod arrays grown by metalorganic chemical vapour deposition. Nanotech. 2005, 16, 2044-2047.
(10) Wang, M.; Hahn, S. H.; Kim, J. S.; Chung, J. S.; Kim, E. J. Koo, K.-K. Solvent-controlled crystallization of zinc oxide nano(micro)disks. J. Cryst. Growth 2008, 310, 1213-1219.

(11) Ranjith, K. S.; Kiruthika, B.; Rajendrakumar, R. T. ZnO microrods to nanowalled microtubes: optimization using simple fluorescence microscopy and enhanced photocatalytic properties. J. Microsc. 2013, 252, 217-225.

(12) Greer, H. F.; Zhou, W. Z.; Liu, M.-H.; Tseng, Y.-H.; Mou, C.$\mathrm{Y}$. The origin of $\mathrm{ZnO}$ twin crystals in bio-inspired synthesis. CrystEngComm 2012, 14, 1247-1255.

(13) Liu, M.-H.; Tseng, Y.-H.; Greer, H. F.; Zhou, W. Z.; Mou, C.$\mathrm{Y}$. Dipole field guided orientated attachment of nanocrystals to twin-brush ZnO mesocrystals. Chem. Eur. J. 2012, 18, 16104-16113.

(14) Zhang, T.; Dong, W.; Keeter-Brewer, M.; Konar, S.; Njabon, R. N.; Tian, Z. R. Site-specific nucleation and growth kinetics in hierarchical nanosyntheses of branched $\mathrm{ZnO}$ crystallites. J. Am. Chem. Soc. 2006, 128, 1096o-10968.

(15) Krishna, K. S.; Mansoori, U.; Selvi, N. R.; Eswaramoorthy, M. Form emerges from formless entities: temperature-induced self-assembly and growth of $\mathrm{ZnO}$ nanoparticles into zeptoliter bowls and troughs. Angew. Chem. Int. Ed. 2007, 46, 5962-5965.

(16) Gao, X.; Li, X.; Gao, W.; Qui, J.; Gan, X.; Wang, C.; Leng, X. Nanocrystalline/nanoporous $\mathrm{ZnO}$ spheres, hexapods and disks transformed from zinc fluorohydroxide, their self-assembly and patterned growth. CrystEngComm 2o11, 13, 4741-4747.

(17) Fang, Y.-J.; Xia, Z. B.; Yu, F. J.; Sha, J.; Wang, Y. W.; Zhou, W. Z. Formation mechanism of hollow microspheres consisting of ZnO nanosheets. CrystEngComm, 1012, 14, 8615-8619.

(18) Karthika, S.; Radhakrishnan, T. K.; Kalaichelvi, P. A review of classical and nonclassical nucleation theories. Cryst. Growth Des. 2016, 16, 6663-6681.

(19) Greer, H. F. Non-classical crystal growth of inorganic and organic materials. Mater. Sci. Technol. 2014, 30, 611-626.

(2o) Self, K.; Zhou, H. J.; Greer, H. F.; Tian, Z. R.; Zhou, W. Z. Reversed crystal growth of $\mathrm{ZnO}$ microdisks. Chem. Commun. 2013, 49, 5411-5413.

(21) Sturm, E. V.; Cölfen, H. Mesocrystals: structural and morphogenetic aspects. Chem. Soc. Rev. 2016, 45, 5821-5833.

(22) Ryan, K. M.; Mastroianni, A.; Stancil, K. A.; Liu, H.; Alivisatos, A. P. Electric-field-assisted assembly of perpendicularly oriented nanorod superlattices. Nano. Lett. 2oo6, 6, 1479-1482.

(23) Liu, Y.; Zhou, L.; Hu, Y.; Guo, C.; Qian, H.; Zhang, F.; Lou, $\mathrm{X}$. W. Magnetic-field induced formation of ${ }_{1} \mathrm{D} \mathrm{Fe} \mathrm{O}_{4} / \mathrm{C} / \mathrm{CdS}$ coaxial nanochains as highly efficient and reusable photocatalysts for water treatment. J. Mater. Chem. 2o11, 21, 18359-18364.

(24) Ahniyaz, A.; Sakamoto, Y.; Bergström, L. Magnetic fieldinduced assembly of oriented superlattices from maghemite nanocubes. Proc. Natl. Acad. Sci. 2007, 104, 17570-17574.

(25) Liu, Z.; Wu, X. D.; Wu, X. L.; Gao, Y. J.; Chen, H. T. Zhu, J. $\mathrm{Chu}, \mathrm{P}$. K. Intrinsic dipole-field-driven mesoscale crystallization of core-shell $\mathrm{ZnO}$ mesocrystal microspheres. J. Am. Chem. Soc. 2009, 131, 9405-9412.

(26) Simon, P.; Zahn, D.; Lichte, H.; Kniep, R. Intrinsic electric dipole fields and the induction of hierarchical form developments in fluorapatite-gelatine nanocomposites: A general principle for morphogenesis of biominerals? Angew. Chem. Int. Ed. 2006, 45, 1911-1915.

(27) Yang, X. F.; Fu, J. X.; Jin, C. J.; Chen, J.; Liang, C. L.; Wu, M. M.; Zhou, W. Z. Formation mechanism of $\mathrm{CaTiO}_{3}$ hollow crystals with different microstructures. J. Am. Chem. Soc. 2010, 132, 1427914287 .

(28) Nicholas, N. J.; Franks, G. V.; Ducker, W. A. Selective adsorption to particular crystal faces of $\mathrm{ZnO}$. Langmuir, 2012, 28, $7189-7196$. 
(29) Herring, N. P.; AbouZeid, K.; Mohamed, M. B.; Pinsk, J.; El-Shall, M. S. Formation mechanisms of gold-zinc oxide hexagonal nanopyramids by heterogeneous nucleation using microwave synthesis. Langmuir 2011, 27, 15146-15154.

(30) Huang, M.; Weng, S.; Wang, B.; Hu, J.; Fu, X.; Liu, P. Various facet tunable $\mathrm{ZnO}$ crystals by a scalable solvothermal synthesis and their facet-dependent photocatalytic activities J. Phys. Chem. C 2014, 118, 25434-25440.

(31) Kaneti, Y. V.; Zhang, Z.; Yue, J.; Zakaria, Q. M. D.; Chen, C.; Jiang, X.; Yu, A. Crystal plane-dependent gas-sensing properties of zinc oxide nanostructures: experimental and theoretical studies. Phys. Chem. Chem. Phys. 2014, 16, 11471-11480.

(32) Matsumoto, K.; Saito, N.; Mitate, T.; Hojo, J.; Inada, M.; Haneda, $\mathrm{H}$. Surface polarity determination of $\mathrm{ZnO}$ spherical particles synthesized via solvothermal route. Cryst. Growth Des. 2009, 9, 5014-5016.

(33) Li, F.; Gong, F.; Xiao, Y.; Zhang, A.; Zhao, J.; Fang, S.; Jai, D. $\mathrm{ZnO}$ twin-spheres exposed in \pm (oo1) facets: stepwise self-assembly growth and anisotropic blue emission. ACS Nano 2013, 7, 10482-10491.

(34) Tian, S.; Li, N.; Zeng, D.; Li, H.; Tang, G.; Pang, A.; Xie, C. Zhao, X. Hierarchical ZnO hollow microspheres with exposed (oo1) facets as promising catalysts for the thermal decomposition of ammonium perchlorate. CrystEngComm 2015, 17, 8689-8696.

(35) Greer, H. F.; Zhou, W. Z.; Zhang, G.; Ménard, H. Nanocone decorated $\mathrm{ZnO}$ microspheres exposing the (ooo1) plane and enhanced photocatalytic properties. Adv. Mater. Interfaces 2017, 4, No. 1601238.

(36) He, K.; Zhao, G.; Han, G. Facile synthesis of novel hierarchical hollow $\mathrm{ZnO}$ microspheres. CrystEngComm, 2014, 16, 3853-3856.

(37) Morkoç, H.; Özgür, Ü. Zinc Oxide: Fundamentals, Materials and Device Technology. Wiley-VCH Verlag GmbH \& Co. KGaA, Weinheim, Germany, 2009, 1-70.

(38) Ostwald, W.; Lehrbruck der Allgemeinen Chemie, Leipzig, Germany, 1896, Vol. 2, Part 1.

(39) Penn, R. L.; Banfield, J. F. Imperfect oriented attachment: Dislocation generation in defect-free nanocrystals. Science 1998, 281, 969-971.

(40) Greer, H. F.; Liu, M.-H.; Mou, C.-Y.; Zhou, W. Z. Dipole field driven morphology evolution in biomimetic vaterite. CrystEngComm 2016, 18, 1585-1599.

(41) Wu, S. T.; Chiang, C.-Y.; Zhou, W. Z. Crystals 2017, 7, 319.

\section{For Table of Contents Use Only}

\section{Formation Mechanisms of ZnO Spherulites and Deritives}

Bethany M. Connolly, Heather F. Greer and Wuzong Zhou

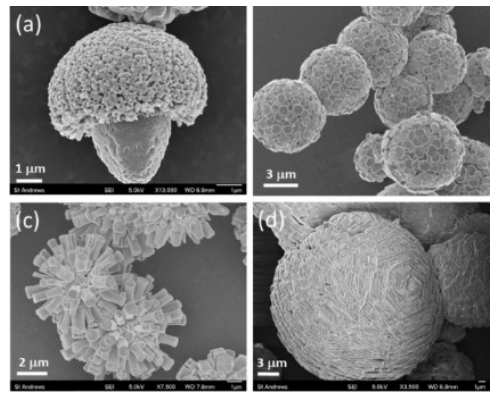

$\mathrm{ZnO}$ microparticles with four different morphologies have been solvothermally synthesized from zinc acetate and formic acid. The formation mechanisms of these morphologies, (a) mushroomlike, (b) microdisc decorated, (c) sea-urchin like and (d) wood gyro-shaped microparticles, via recrystallization of microspherulites are investigated. It is proposed that dipole field force plays an important role in these morphology evolution processes. 